\title{
Epidural blood patch for post dural puncture headache in a parturient with multiple sclerosis
}

\section{Mihály Kovács MD', Manoj Ravindran FRCA'}

'Consultant Anaesthetist, Department of Anaesthesia, George Eliot Hospital, Nuneaton, UK

Disclosures:The authors have no conflicts of interest to report

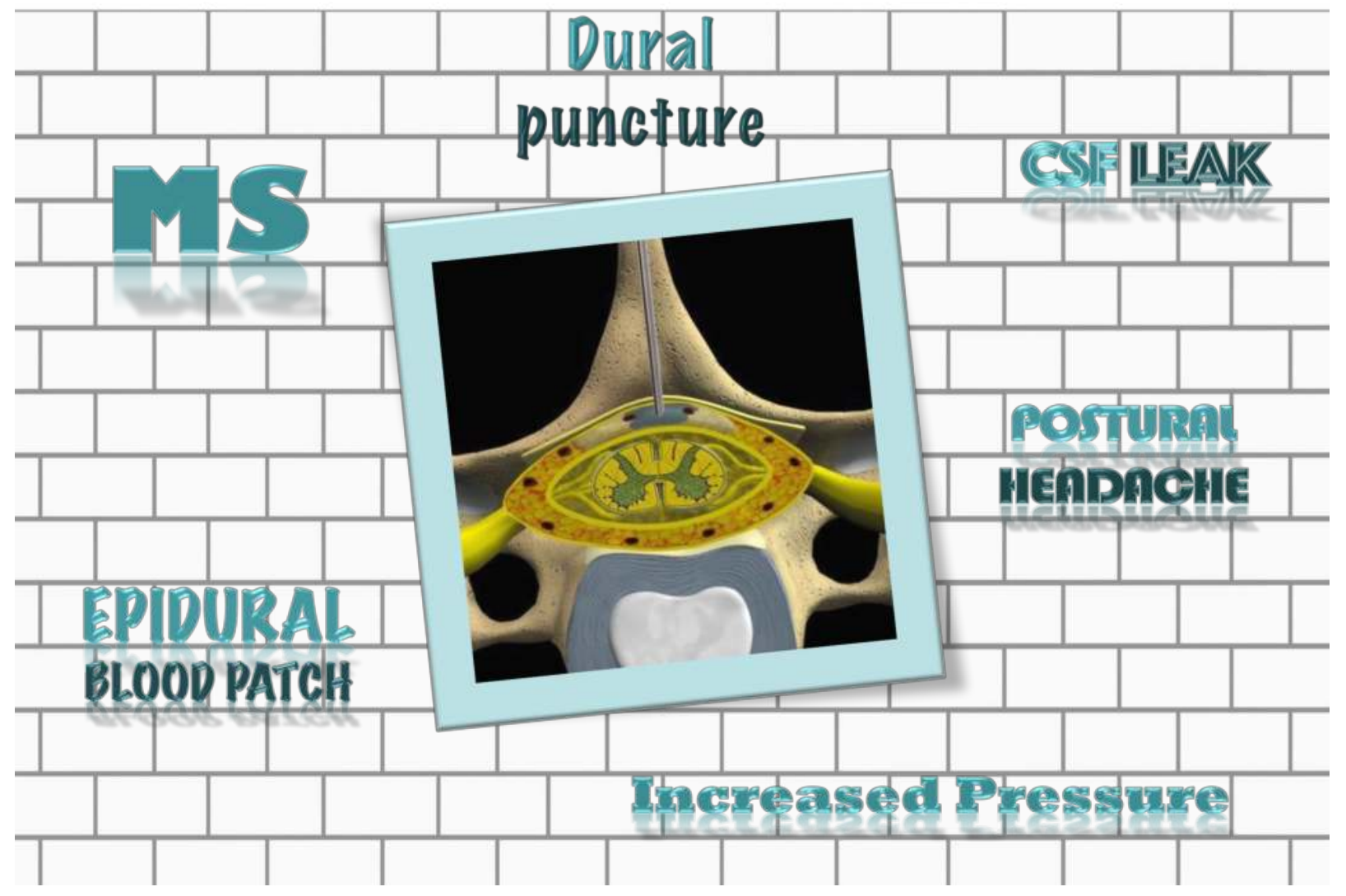

\section{Background}

Multiple sclerosis (MS) is a serious chronic neurological disorder in which demyelination, axonal injury and inflammation occur in the white matter of the central nervous system with formation of sclerotic plaques and general sparing of the peripheral nerves. Nevertheless, after careful consideration, patients with MS may receive neuraxial anaesthesia. A proportion of these patients might suffer from one of the most common side effects of these blocks: post dural puncture headache (PDPH). The gold standard treatment of this condition, when conservative management has failed is epidural blood patch (EBP). However there is a considerable lack of evidence regarding the safety and efficacy of EBP in MS patients.

One of the main concerns with this technique is the consequently increased epidural pressure, that can interfere with the conduction of axons affected by MS.' In our report we describe a rare case of a successful EBP treatment of PDPH in a patient diagnosed with MS.

\section{Case report}

A 32 year old primipara presented on labour ward with a past medical history of vitamin BI2 deficiency and relapsing-remitting MS. Her MS was diagnosed in 2015 and initial symptoms were blurred and double vision with poor balance. MRI of the brain was in keeping with demyelination. She has had one relapse with reduced balance since then. She has not had any difficulties with strength, sensation or cognition. She had a lumbar puncture in the past, and she developed PDPH that lasted for seven days and was treated conservatively. During pregnancy she was without any neurological symptoms, and she was not on any medication. She was seen in pre-assessment anaesthetic clinic and was given the option of having epidural analgesia for labour. Soon after admission to labour ward, she requested labour epidural for pain relief. The procedure was successful at first attempt at L3-L4 interspace, with no obvious signs of dural tap through needle or catheter. Midwifes reported a good epidural analgesia, and she has successfully delivered her baby. It is worth mentioning that the unintentional dural puncture could have contributed to the excellent and prompt pain relief.

\author{
WHS \\ George Eliot Hospital \\ NHS Trust
}

24 hours after epidural insertion patient developed a postural, frontooccipital PDPH. She was prescribed regular pain relief as per our protocol. On the 2nd postpartum day, she still had severe symptoms from the PDPH, and we decided to perform an EBP in theatre.

After gaining full informed consent from the patient, EBP was performed in left lateral position under full asepsis. The same interspace used for initial epidural was identified. $30 \mathrm{mls}$ of autologus blood was then obtained from the anticubital fossa under full asepsis and the blood injected slowly, over 2-3 minutes. The patient did not report any neurological symptoms during the procedure, and only reported mild pressure in her back at the end of injection. Peridural pressure monitoring or somatosensory evoked response measurment was not available on site.

After 3 hours of bedrest PDPH symptoms were permanently relieved. She was follwed up after the procedure in the anaesthetic clinic and has not had any headache or any motor or sensory function deterioration post-EBP.

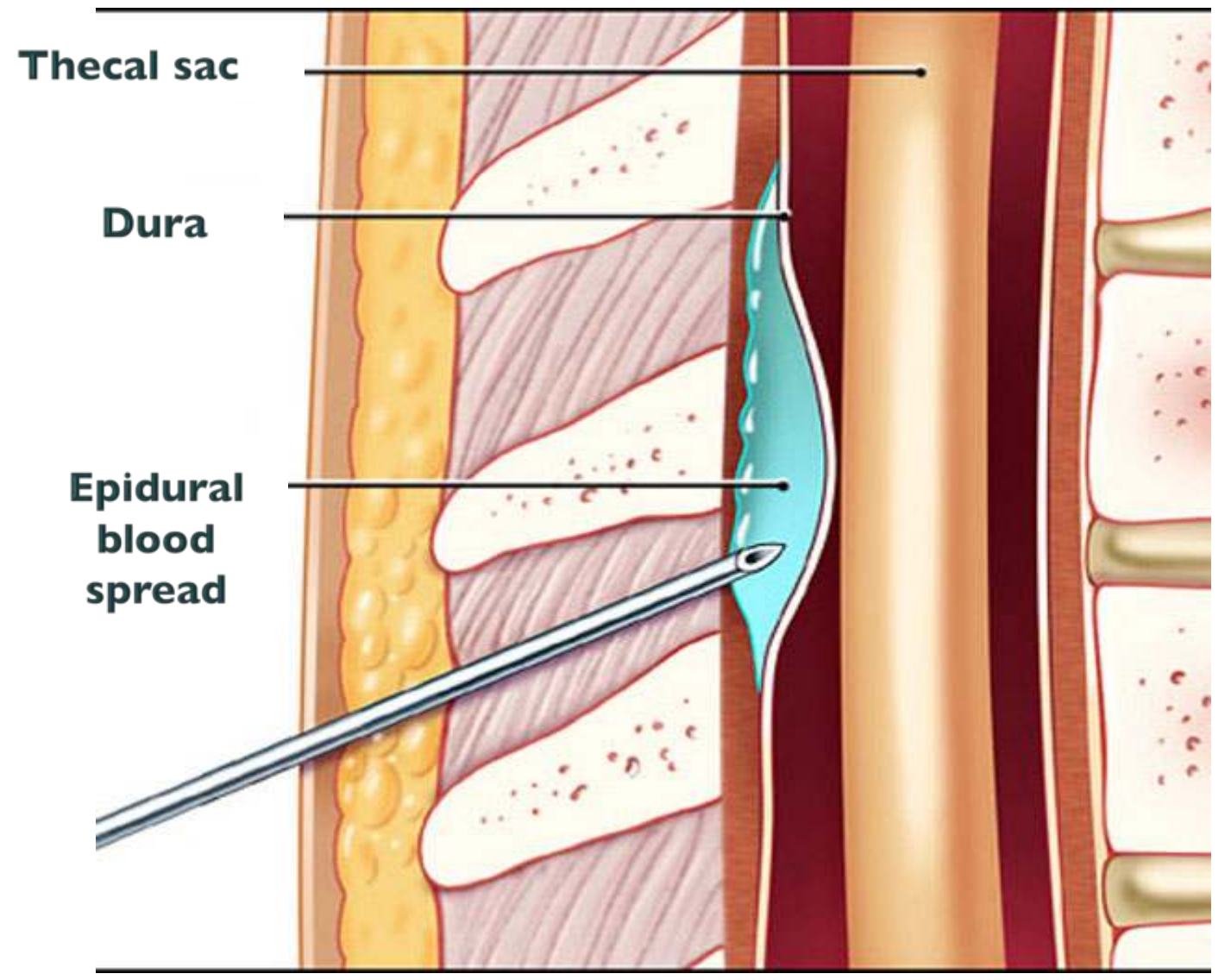

\section{Discussion}

We have presented a case of a successful treatment of PDPH with EBP in a MS patient. The way of performing EBP in MS has only been reported once. ${ }^{3}$ The main concern with EBP is the consequently increased epidural pressure, that may interfere with the conduction of axons affected by MS.' Though, epidural pressure rise can be reduced by slower injection of fluid. ${ }^{4}$ Our case demonstrates, that EBP can be safe in MS, with a rapid improvement of PDPH. Literature suggests that somatosensory evoked response monitoring should be considered when performing EBP in MS patients to quantify interference with axonal conduction. ${ }^{3}$ However, this might not be available at every hospital as seen in our example.

\section{Learning point}

EBP can be a safe treatment option of PDPH in MS patients when injection is performed at a slow rate.

\section{Aknowledgments}

George Eliot Hospital NHS Trust

\section{References:}

I.Vercauteren M, Heytens L. Anaesthetic considerations for patients with a pre-existing neurological deficit: are neuraxial techniques safe? Acta Anaesthesiol Scand 2007; 51:83I-8.

2. https://mmcneuro.wordpress.com/headache-and-abnormal-brain-mri-whats-the-diagnosis-answer/

3. Koeva V, Bar-Or A, Gendron D, Backman SB. Epidural blood patch in a patient with multiple sclerosis: is it safe? J Can Anesth 201 3;60:479-483.

4. Riffel B, Stohr M, Korner S. Spinal and cortical evoked potentials following stimulation of the posterior tibial nerve in the diagnosis and

localization of spinal cord diseases. Electroencephalogr Clin Neurophysiol 1984; 58:400-7. 